Proceedings of the 16th Czech and Slovak Conference on Magnetism, Košice, Slovakia, June 13-17, 2016

\title{
Low Magnetic Field Response in Ferronematics
}

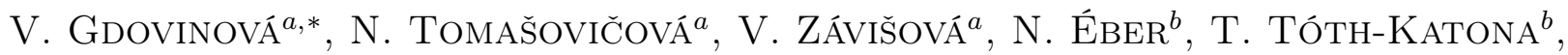 \\ F. ROYER ${ }^{c}$, D. JAMON ${ }^{c}, J$. JADZYN ${ }^{d}$ AND P. KOPČANSKÝ ${ }^{a}$ \\ ${ }^{a}$ Institute of Experimental Physics, SAS, Watsonova 47, 04001 Košice, Slovakia \\ ${ }^{b}$ Institute for Solid State Physics and Optics, Wigner Research Centre for Physics, Hungarian Academy of Sciences, \\ H-1525 Budapest, Hungary \\ ${ }^{c}$ Université de Saint Etienne, Saint Etienne, France \\ ${ }^{d}$ Institute of Molecular Physics, Polish Academy of Sciences, M. Smoluchowskiego 17, 60-179 Poznań, Poland
}

\begin{abstract}
In this work the 4- $n$-hexyl-4'-cyanobiphenyl liquid crystal was doped with differently shaped magnetite nanoparticles. The structural changes were observed by capacitance measurements. Influence of the shape of magnetic particles on magnetic Fréedericksz transition depends on the type of anchoring, which is characterized by the density of the anchoring energy and by the initial orientation between the liquid crystal molecules and the magnetic moment of the magnetic particles. It was observed that in the case of doping with spherical particles, the critical magnetic field is shifted to higher values with increase of volume concentration of the magnetic nanoparticles but decreases with increase of biasing voltage. In the case of doping with rod-like particles, the critical magnetic field is almost independent of the volume concentration of the magnetic nanoparticles.
\end{abstract}

DOI: 10.12693/APhysPolA.131.934

PACS/topics: 75.50.Mm, 61.30.Gd, 61.30.Hn

\section{Introduction}

Liquid crystals (LCs) belong to a class of soft condensed matter that is characterized by the combination of the fluidity of ordinary liquids with the direction dependent electric and optical properties of crystalline solids. For a long time, LCs have remained a pure scientific curiosity and have not attracted great attention among scientists. Nowadays LCs are best known for their successful application in flat panel displays, but they exhibit many unique and attractive properties that offer great potential for fundamental science and innovative applications [1]. One of the most important early findings related to LCs was that they can be controlled by external fields. Their reorientational response typically has a threshold behaviour - an effect described by Fréedericksz [2] and named after him as the Fréedericksz transition. LCs can be (re)oriented with electric or magnetic fields due to the anisotropy of their dielectric permittivity $\left(\epsilon_{a}\right)$ or diamagnetic susceptibility $\left(\chi_{a}\right)$, respectively. The dielectric anisotropy of liquid crystalline materials is of the order of unity, therefore the required driving voltages are of the order of a few volts. LCs are less sensitive to the magnetic field, due to their very low anisotropy of the diamagnetic susceptibility $\left(\chi_{a} \approx 10^{-7}\right)$. As a result of this, the realignment of LCs may require a large magnetic field strength $H$ with $B=\mu_{0} H$ being of the order of $1 \mathrm{~T}$ ( $\mu_{0}$ is the permeability of vacuum) [3]. The idea of doping LCs by fine magnetic particles (MPs) that may enhance their sensitivity to magnetic field, comes from Brochard and de Gennes [4].

\footnotetext{
*corresponding author; e-mail: gdovinova@saske.sk
}

Ferronematics (FNs) represent stable colloidal suspensions of fine MPs in nematic LCs that can be easily aligned by a magnetic field and therefore they act as nanomagnets. The most essential feature of FNs is a strong coupling between the MPs (their magnetic moment $\boldsymbol{m}$ ) and the LC matrix (the director $\boldsymbol{n}$ ). This coupling ensures that the effect of magnetic field will be transferred into the nematic host. The Brochardde Gennes theory [4] predicted a rigid anchoring with $\boldsymbol{m} \| \boldsymbol{n}$. Later experiments on some thermotropic ferronematics have indicated that besides the $\boldsymbol{m} \| \boldsymbol{n}$ condition, the case of $\boldsymbol{m} \perp \boldsymbol{n}$ is also possible. Based on these experiments, Burylov and Raikher modified the theoretical description of FNs $[5,6]$. They considered a finite value of the surface density of the anchoring energy $W$ at the nematic-magnetic particle boundary and defined a parameter $\omega$ as the ratio of the anchoring energy to the elastic energy of the LC $(\omega=W d / K$, where $d$ is the size of the MPs and $K$ represents an orientational-elastic Frank modulus). The parameter $\omega$ defines the type of the anchoring of nematic molecules on the MP's surfaces. Rigid anchoring is present at $\omega \gg 1$, while soft anchoring is characterized by $\omega \leq 1$; this latter type of anchoring permits both types of boundary conditions ( $\boldsymbol{m} \perp \boldsymbol{n}$ and $\boldsymbol{m} \| \boldsymbol{n})$.

The present paper discusses experimental observations on the structural transitions in FNs based on the liquid crystal 4- $n$-hexyl-4'-cyanobiphenyl (6CB) doped with magnetic nanoparticles of different size and shape. The dielectric properties of prepared FNs have been investigated during application of orienting magnetic $(H)$ and dc biasing electric $\left(E_{\text {bias }}\right)$ fields.

\section{Materials and methods}

FN samples were based on the thermotropic nematic $6 \mathrm{CB}$, which has high chemical stability and a conve- 
nient temperature range of the nematic phase [7]. The LC was doped with magnetic particles (MPs) of various shapes (spherical and rod-like) in low volume concentrations $\left(\phi_{1}=10^{-5}\right.$ and $\left.\phi_{2}=10^{-4}\right)$. The spherical MPs were prepared by the co-precipitation method described in [8]. The rod-like MPs were synthesized through hydrolysis of $\mathrm{FeCl}_{3}$ and $\mathrm{FeSO}_{4}$ solutions containing urea [9]. The morphology and size distribution of the prepared nanoparticles were determined by transmission electron microscopy (TEM) [10]. The mean diameter of spherical MPs was $d=10 \mathrm{~nm}$, while for rod-like particles $d=11 \mathrm{~nm}$ and their mean length was $L=240 \mathrm{~nm}$. The structural transitions in ferronematic samples have been monitored by capacitance measurements in a capacitor made of indium-tin-oxide (ITO) coated glass electrode. The capacitor with the electrode area of approximately $1 \mathrm{~cm} \times 1 \mathrm{~cm}$ has been placed into a thermostat system, regulated with a temperature stability of $0.05^{\circ} \mathrm{C}$. The distance between the electrodes (the sample thickness) was $D=50 \mu \mathrm{m}$. The capacitance $C$ was measured at the frequency of $1 \mathrm{kHz}$ by a TiePie Handyscope HS5 instrument, using sinusoidal voltage signal with the amplitude of $0.1 \mathrm{~V}$ that was switched to the cell.

\section{Results and discussion}

The structural transitions provoked by combined electric and magnetic fields were monitored. The ac testing $\left(U_{t}\right)$ and the dc biasing $\left(U_{\text {bias }}\right)$ electric voltages, as well as the magnetic field $H$ were applied perpendicular to the initial director orientation. As the electric and magnetic fields are parallel, due to the same sign of $\epsilon_{a}$ and $\chi_{a}$, they induce the same type of distortion. Figures 1 and 2 show how the reduced capacitance of the undoped $6 \mathrm{CB}$ and $6 \mathrm{CB}$ doped with rod-like and spherical MPs, respectively, depend on the external magnetic field $H$, measured at no bias voltage $\left(U_{\text {bias }}=0 \mathrm{~V}\right)$. For the LC doped with spherical MPs, doping increased the critical magnetic field $H_{c}$, whereas rod-like MPs did not shift $H_{c}$ significantly.

Figures 3 and 4 show how the critical magnetic fields of pure $6 \mathrm{CB}$ and of $6 \mathrm{CB}$ based FNs depend on the bias voltage. In case of doping with rod-like particles, the critical

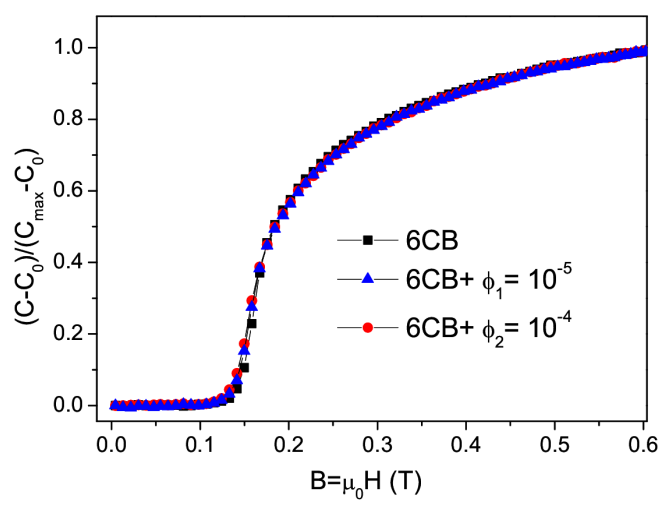

Fig. 1. Dependence of the reduced capacitance on the external magnetic field for undoped $6 \mathrm{CB}$ and for $6 \mathrm{CB}$ doped with rod-like particles in two concentrations $\phi$.

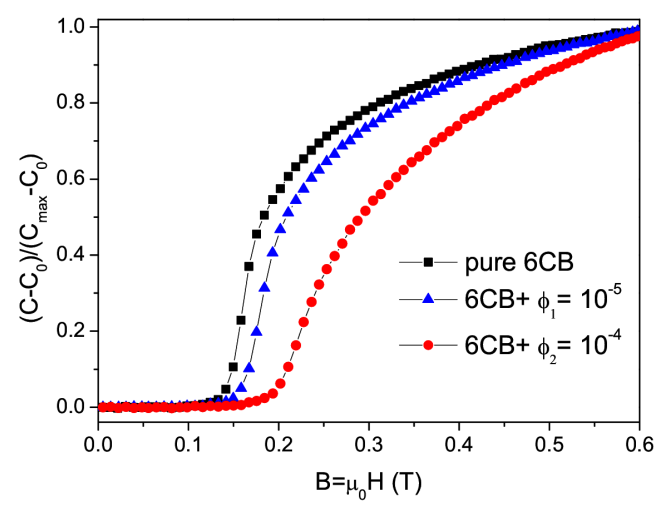

Fig. 2. Dependence of the reduced capacitance on the external magnetic field for undoped $6 \mathrm{CB}$ and for $6 \mathrm{CB}$ doped with spherical particles in two concentrations $\phi$.

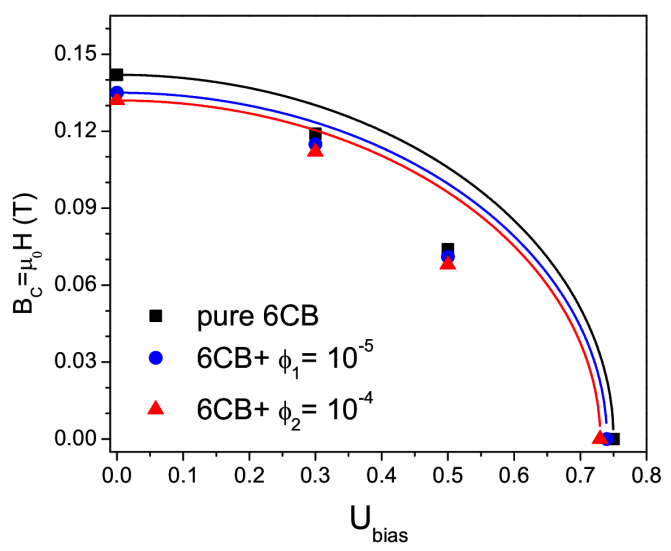

Fig. 3. Dependence of the critical magnetic field $H_{c}$ on the bias voltage $U_{\text {bias }}$ for the pure $6 \mathrm{CB}$ and for $6 \mathrm{CB}$ doped with rod-like magnetic particles. Solid lines represent theoretical calculation according to Eq. (1).

magnetic field is almost independent of the volume concentration of MPs (Fig. 3). However, when the doping is with spherical particles, the critical magnetic field is shifted to higher values with increase of volume concentration of MPs (Fig. 4). In both cases, $H_{c}$ decreases with the increase of the dc bias voltage $U_{\text {bias }}$, as it is expected. However, as one sees from Figs. 3 and 4, the decrease is much more pronounced than it follows from the relation for the combined magnetic and electric field action:

$$
\left(\frac{H}{H_{c}}\right)^{2}+\left(\frac{U}{U_{c}}\right)^{2}=1
$$

For the interpretation of these results one has to recall the Burylov-Raikher theory. From the expression for the free energy of FNs, they have derived an approximate relation between the critical field $H_{c}$ of the nematic host and the critical field $H_{C F N}$ of the FNs [6]:

$$
H_{C F N}^{2}-H_{c}^{2}=\mp \frac{2 W \phi}{\mu_{0} \chi_{a} d} .
$$

Here $d$ is the mean diameter of the MPs and $\phi$ is their volume concentration. The sign depends on the initial orientation of $\boldsymbol{m}$ and $\boldsymbol{n}$. As both $H_{C F N}$ and $H_{c}$ were 


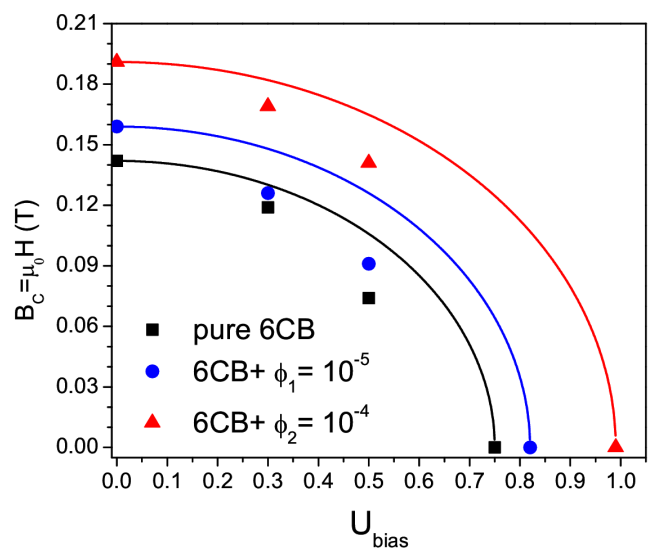

Fig. 4. Dependence of the critical magnetic field $H_{c}$ on the bias voltage $U_{\text {bias }}$ for the pure $6 \mathrm{CB}$ and for $6 \mathrm{CB}$ doped with spherical magnetic particles. Solid lines represent theoretical calculation according to Eq. (1).

measured, Eq. (2) can be used to estimate the anchoring energy density $W$ at the nematic-magnetic particle boundary. Using the data at $U_{\text {bias }}=0$ and the value of $\chi_{a}=1.078 \times 10^{-6}$ for $6 \mathrm{CB}$ (at $T=25^{\circ} \mathrm{C}$ ) and the volume concentration $\phi_{2}=10^{-4}$ of the MPs, one obtains $W \approx 10^{-8} \mathrm{~N} \mathrm{~m}^{-1}$ for the rod-like MPs and $W \approx 10^{-7} \mathrm{~N} \mathrm{~m}^{-1}$ for the spherical ones. Now the parameter $\omega$ can also be calculated. As for $6 \mathrm{CB}$ at the measuring temperature $K_{1}=4.4 \mathrm{pN}, \omega \approx 10^{-4} \ll 1$ is obtained for rod-like magnetic particles and $\omega \approx 10^{-3} \ll 1$ characterizes the spherical MPs. These values correspond to a soft anchoring of the nematic director on the surfaces of the magnetic particles in 6CB-based FNs. We note that in a previous work [8], the influence of the shape of magnetic particles on the structural transitions in another LC, 4-(trans-4'-n-hexylcyclohexyl)isothiocyanatobenzene (6CHBT), has also been studied. Those experimental results concluded in soft anchoring in the case of spherical MPs, but in a rigid one in the case of rod-like or chain-like MPs, all with $\boldsymbol{m} \| \boldsymbol{n}$ initial orientation. These results differ from the present findings. Thus we can conclude that the type of the anchoring of nematic molecules on the MP's surfaces depends on the shape of the MPs as well as on the type of the host LC.

\section{Conclusions}

The influence of the shape of magnetic particles on the critical magnetic field was studied in 6CB-based FNs, whose behaviour differed from that of 6CHBT-based FNs. It was demonstrated that addition of the nanoparticles has a substantial influence on the sensitivity of FNs to external magnetic fields. Our experimental results indicated soft anchoring in the cases of spherical as well as of rod-like magnetic particles. Doping with spherical MPs increased the critical field of the magnetic Freedericksz transition, which indicates an initial $\boldsymbol{m} \perp \boldsymbol{n}$ orientation. In the case of doping with rod-like MPs, $B_{c}$ shifts almost negligibly, but to opposite direction. As a result the initial orientation permits either parallel or perpendicular orientation between the magnetic moment of MPs and the director.

\section{Acknowledgments}

This work was supported by projects VEGA $2 / 0045 / 13$, the Slovak Research and Development Agency under the contract No. APVV-0171-10 and APVV-SK-HU-2013-0009, Ministry of Education Agency for Structural Funds of EU in frame of project 26220120033 and 26220120021, EU FP7 M-era.Net MACOSYS (Hungarian Scientific Research Fund OTKA NN 110672), and the Hungarian National Research, Development and Innovation Office (NKFIH) grant TÉT 12 SK-1-2013-002.

\section{References}

[1] J.P.F. Lagerwall, G. Scalia, Curr. Appl. Phys. 12, 1387 (2012).

[2] V. Freedericksz, V. Zolina, Trans. Faraday Soc. 29, 919 (1970).

[3] P.G. de Gennes, The Physics of Liquid Crystals, Clarendon Press, Oxford 1974.

[4] F. Brochard, P.G. de Gennes, J. Phys. (France) 31, 691 (1970).

[5] S.V. Burylov, Y.L. Raikher, Phys. Lett. A 149, 279 (1990).

[6] S.V. Burylov, Y.L. Raikher, Mol. Cryst. Liq. Cryst. 258, 123 (1995).

[7] J. Czub, S. Urban, A. Wurflinger, Liq. Cryst. 33, 85 (2006).

[8] P. Kopčanský, N. Tomašovičová, M. Koneracká, V. Závišová, M. Timko, A. Džarová, A. Šprincová, N. Éber, K. Fodor-Csorba, T. Tóth-Katona, A. Vajda, J. Jadzyn, Phys. Rev. E 78, 011702 (2008).

[9] N. Tomašovičová, P. Kopčanský, N. Éber, in: Anisotropy Research: New Developments, Ed. H.G. Lemu, Hauppauge, Nova Science, New York 2012, Ch. 11, p. 245.

[10] V. Gdovinová, N. Tomašovičová, N. Éber, T. TóthKatona, V. Závišová, M. Timko, P. Kopčanský, Liq. Cryst. 41, 1773 (2014). 